\title{
Information Management for Data Retrieval in a Picture Archive and Communication System
}

\author{
Fred W. Prior and Kamal H. Nabijee
}

\begin{abstract}
Data stored in a Picture archive and communication system (PACS) must be organized to permit efficient retrieval. The concept of a unique data object identifier (UID) permits a fundamental partitioning of the problem into a storage system, indexed by UID, and a database containing descriptive elements. The database serves to map user retrieval requests, expressed in terms of clinically relevant descriptive elements, and into UIDs of specific data objects. Different data organization mechanisms are employed by imaging modalities, thereby making the structure of a generic PACS database complex. One solution may be derived by analogy from film-based systems. Images and other data objects may be organized, by application of modality and site specific rules, into electronic folders. Folders may then be organized into a patient master folder or userdefined reference folders. Such an organization provides an easily understood user access model for information stored in PACS archives. This report presents a PACS architecture comprised of an Information Management System (IMS) and Information Storage System (ISS). Entity-relationship diagrams are presented to define a schema for the IMS database, based on the folder analogy. The folder concept and its relationship to the ACR-NEMA Standard are discussed.

(C) 1989 by W.B. Saunders Company.
\end{abstract}

KEY WORDS: PACS, archive, information management system, image retrieval.

$\mathbf{A}^{\mathrm{r}}$ PRIMARY motivation for the development of picture archive and communication systems (PACS) has been the replacement of conventional, film-based medical image handling systems with digital, electronic systems. Most development to date has focused on workstations, communications standards, and networking. It is assumed that high-density optical storage techniques are, or soon will be, available to provide permanent archival storage. One question that seems to have been largely overlooked, however,

From the PACS Engineering Department, Siemens Gammasonics Inc, Des Plaines, IL.

Presented at the Society of Photo-Optical Instrumentation Engineers meeting, Newport Beach, RI, 1989.

Address reprint requests to Fred $W$. Prior, MS, PACS

Engineering, Siemens Gammasonics Inc, 2000 Nuclear Dr,

Des Plaines, IL 60018.

(- 1989 by W.B. Saunders Company.

0897-1889/89/0203-0001\$03.00/0 is the location and retrieval of a particular set of images once they have been stored in a PACS.

Current digital modalities have fairly simple directory structures to organize information on local storage. Most systems rely on a default temporal sorting (eg, only the images made today for a particular patient are on the system). Older images may have been archived to magnetic tape or even optical disk, but the task of locating these usually involves a manual search of paper records.

Archival storage is, of course, currently based on film. Many mechanisms exist for organizing and tracking films. Some involve a computerized database. Ultimately, however, film archives require one to manually search a file room for the films associated with a particular patient name or access code number. In a digital system, it is not intuitively obvious how one performs the function equivalent to walking through the stacks of a film archive.

The volume of image data generated annually by a fully digital radiology department has been estimated by several authors. ${ }^{1,2}$ Most estimates suggest generation rates of several hundred thousand images annually; thus requiring several Terabytes of storage. Legal requirements in many areas and for some specialities (eg, pediatrics) require this data to be kept and retrievable for up to 25 years. With this volume of data, browsing a directory listing to locate a particular patient or a single image is not an attractive proposition.

An obvious approach for tracking digital images in PACS is a database management system. Many commercial products exist and have found wide application. The theory and technology, at least in the case of relational systems, is relatively mature. ${ }^{3}$ Unfortunately, simply having a database management system does not solve the image location and retrieval problem. The real question is, how to structure the database to make retrieval efficient and flexible given the potential for massive growth.

An approach that seems promising may be derived by analogy to existing film based systems. Films are associated with one another, and with other relevant information (eg, reports, 
laboratory data) by placing them into a cardboard folder. Folders are organized in the film room by placing them in a patient's master jacket. There are obviously many specific variations of this nesting of data envelopes but the use of a folder as the primary means of organization is fundamental. It will be shown that there are actually several very useful electronic analogs of the folder concept.

\section{UNIQUE DATA OBJECT IDENTIFIER}

$A$ basic and very powerful concept in computer science is the distinction between a datum and a reference to it. Such reference elements are called pointers. Modern software languages support complex manipulations of pointers. By using pointers, it is possible to answer questions concerning the existence of a datum and control access to it without physically interacting with the data. The pointer can, in many instances, take the place of actual data. Manipulation of pointers is usually much more efficient than manipulation of the data they reference.

In a database, the only record level addressing mechanism available is the "primary key." It uniquely identifies a record at the logical level of the database structure. Relationships between entities are formed by association of their primary key attributes.

If one defines a unique data object identifier (UID) such that it identifies a particular data object in all PACS, it can serve both as a pointer to its associated data object and as a primary key to locate descriptive information about the data object in a database. The structure of a UID must be such that it applies universally across all equipment types, manufacturers, and geographic regions. For simplicity it should be defined as a single data element (ie, a single string or array).

The digital imaging and communications standard jointly developed by the American College of Radiology (ACR) and the National Electrical Manufacturers Assocfiation (NEMA) ${ }^{4}$ provides a common data dictionary to facilitate the interpretation of medical information passed across a communication interface. This dictionary is obviously the first place to look for the definition of a UID. Unfortunately, the standard contains no mechanism for uniquely identifying data objects (within the ACR-NEMA standard the term Data Set is used to describe a collection of data elements that are transmitted as a single message. The term Data Object as used here is essentially synonomous with Data Set except that it is defined outside the context of the communication interface, i.e. it may also be a storage structure). It suggests, but does not require, that the elements Station Id, Study Date, Study, Series, Acquisition, and Image can be concatenated to produce a unique identifier of the form "Study $\backslash$ Series $\backslash$ Acquisition $\backslash$ Image." The standard suggests that this type of identifier can be used to describe the source images used to create a composite or collage data object, and to provide an audit trail for modified data objects.

The limitations of this approach are obvious. The recommended identifier string is quite complex and its structure is not fixed. It is also not necessarily unique across systems. No attempt is made in the ACR-NEMA standard to define a unique identifier for any data objects other than images.

A more appropriate solution has been proposed as part of the Standard Product Interconnect (SPI) extensions to ACR-NEMA. ${ }^{5}$ Within SPI, a UID is defined as a single data element that is comprised of a 26 character ASCII string with the following format:

$$
\begin{aligned}
\text { UID }=<\text { SYSTEM } & \text { ID } \\
& <<\text { IE UNIT }> \\
& <\text { DATE-TIME STAMP }>
\end{aligned}
$$

the symbols $<>$ indicate string concatenation. The SYSTEM ID is a six character string that consists of a three-character CCITT country code and a three-character manufacturer code and system number. The IE UNIT consists of a two-character modality code and a two-character unit number. The DATE-TIME STAMP is the concatenation of the date and time of creation of the data object, expressed in ACRNEMA-defined format (the time is only recorded to the nearest hundredth of a second and punctuation marks are supressed).

This definition of a UID applies to any type of data object and can deal with data objects that have been transported across geographic, manufacturer, and modality boundaries. The SPI format UID can uniquely identify a data object in all PACS systems, assuming that there is no image source that can generate images faster than one every ten milliseconds. 


\section{DISTRIBUTED STORAGE SYSTEMS}

The SPI defines a data object somewhat more precisely than the loose definition given above. A data object is the concatenation of three disjoint sets of data elements: the UID, the data object description (DESC), and the remaining elements as required by ACR-NEMA (including the image or text data elements). The DESC is defined as the set of data elements expected to be queried for data object selection. The DESC set is a subset of the elements specified by the ACRNEMA standard to be associated with a particular type of data object.

The definition of DESC and UID permits a fundamental partitioning of the PACS storage and retrieval problem into two related but separable components: a database composed of DESC elements over which query operations are defined, and a simple bulk storage component containing the actual data objects indexed by UID. Based on this primary partitioning, the data storage and retrieval problem can be solved by creating two classes of components: an information management system (IMS) to manage descriptive information and an information storage system (ISS) to manage data objects (Fig 1).

The IMS processes query and update transactions and acts much like a card catalog in a library. It must remember the location of every data object that has been submitted to the PACS. The IMS database has to provide a cross-reference for each data object that permits PACS users to search for data objects using keys that are relevant to their specific application (eg, patient name, modality type, date, organ system, etc).

Ultimately, all data retrieval queries result in the requester being provided a unique data object pointer that consists of the UID and a location

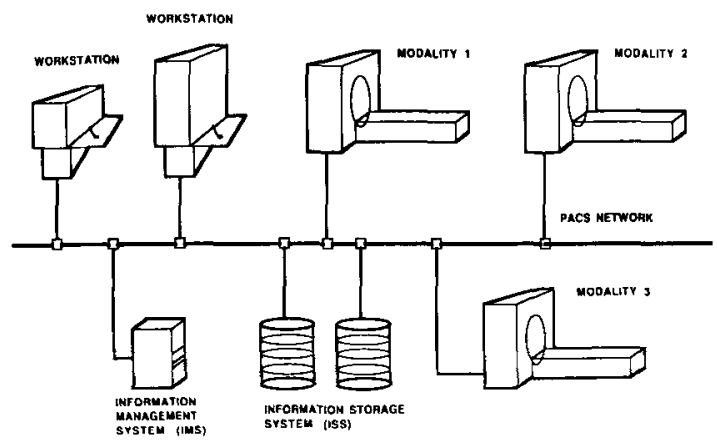

Fig 1. PACS architecture. (ie, the logical name of the node which is storing that data object). These two pieces of information, UID and location, provide the linkage between the IMS and the ISS.

The ISS is a large, distributed bulk storage bin. It must support a simple directory function to map UIDs into internal storage addresses. The ISS may be subdivided into STORE and ARCHIVE type nodes, based on whether the storage is temporary (high-speed magnetic media) or permanent (large volume optical media).

This distributed storage architecture simplifies the design of the logical components needed for PACS data storage and retrieval. ISS components are simple file servers that require only a UID to access data. The number of such servers on a PACS is restricted only by cost and bandwidth considerations. All database functions are localized to a single directory server (IMS). To provide an unambiguous target for query and update operations, a logical PACS can contain only one such server.

Based on the distributed storage architecture, a PACS can be defined as a logical network that incorporates exactly one IMS function and at least one ISS function. Each PACS is autonomous in that the local IMS performs all functions of a PACS and knows about all data objects contained in the ISS components of that PACS. Although at first glance this architecture may seem somewhat restrictive, it is capable of almost unlimited growth since each PACS, with all its functionalities, can be treated as a single module or "island" that can be connected to other PACS. ${ }^{6}$

\section{THE DISPARATE DIRECTORY PROBLEM}

In a system based on the distributed storage architecture, the internal structure of ISS components is fairly obvious, but the structure of the IMS database is not. All medical imaging modality systems provide some mechanism to organize sets of images into

tions. Unfortunately the rules and parameters for forming such associations are specific to each modality and manufacturer. A PACS database server must somehow normalize the modalityspecific association rules into some generic structure that will permit users of multimodality workstations to retrieve desired sets of data.

An examination of the rules of association applied by a cross-section of modality systems 
reveals at least two basic classes: demographic and display. To clarify the distinction, a particular set of image data objects may represent a GI series performed for a particular patient on a specific date. The authors classify this type of association as demographic. On the contrary, a different set of image data objects (or perhaps the same set), taken in a particular order, form a cine loop. This is what is meant by a display association. Descriptions of both types of associations are normally contained in the directory of a modality system. Both types of information must somehow be communicated to and understood by PACS workstations and database servers.

The ACR-NEMA standard attempts to address the communication of association information (the standard is not concerned with interpretation or display of information) by defining a generic directory hierarchy into which any modality directory structure can be mapped. The elements Study, Series, Acquisition, and Image form the basis of this hierarchical association mechanism. One will note that the same set of elements have been used in two different contexts. In fact, the ACR-NEMA standard does not recognize the distinction between data object association rules and unique data object identification although version 2.0 of the ACR-NEMA standard contains an explicit acknowledgment of this distinction.

It can be argued that any modality directory structure can be mapped into the Study, Series, Acquisition, and Image hierarchy. The critical question is, once this has been done, will a user of a PACS workstation be able to understand the association. For example, one digital subtraction angiography sytem (DIGITRON, Siemens Medical Systems, Iselin, NJ) has a directory hierarchy of the form Patient, Study, Image, and Frame. While it is true that it is possible to map this to the ACR-NEMA hierarchy, the redefinition of the term Image will undoubtedly cause confusion (an ACR-NEMA image is equivalent to a Frame).

If it is assumed that images are communicated as separate data objects, including the association, information within each data object is awkward. This is especially true if one is trying to describe both the demographic association and one or more display associations among the same set of data objects. Clearly what is needed is a mechanism to describe several different associa- tions among data objects and is at least partially external to those data objects.

\section{THE FOLDER ANALOGY}

If one observes the organization and flow of data in a radiology department, it is obvious that a primary mechanism for associating and transporting information is a folder or jacket. The films resulting from an examination are placed into a folder and the folder is transported to the reading room. When a report has been generated, a paper copy is added to the folder. Demographic, diagnostic, and treatment-related information is often included as well. Finally, the folder is added to the patient's master jacket in the film library. While this may be an oversimplification of the functioning of a radiology department, it illustrates the utility of the folder as a data envelope. The obvious question is, can one define an electronic analog of the manila folder?

Many personal computer users are familiar with one representation of an electronic folder. There are numerous ways to graphically represent the concept of a folder (eg, an icon, a formatted directory listing, or a set of images arranged on a workstation display). Such representations may be thought of as a display metaphor that allows a user to visualize and manipulate electronic folders.

In general, an electronic folder is a data structure that contains other data structures either by value or, more frequently, by reference (the machine representation of a display icon is, in fact, a data structure). One can think of a folder as a file on a computer system. This file may contain pointers to other files, some of which may themselves be folders. This type of nested data structure is often used to implement a directory service in mini- and microcomputer systems.

Figure 2 illustrates four representations or incarnations of the folder concept. The folder as a display metaphor and as a directory structure may find important applications in PACS workstations, but they are not particularly germane to the present discussion. There are two other incarnations of the folder concept, however, that are extremely important: the folder as a data object and as a database entity.

The SPI defines a data object of type folder. Like other SPI data objects, a folder consists of a UID, a DESC, and other elements. Unlike other 


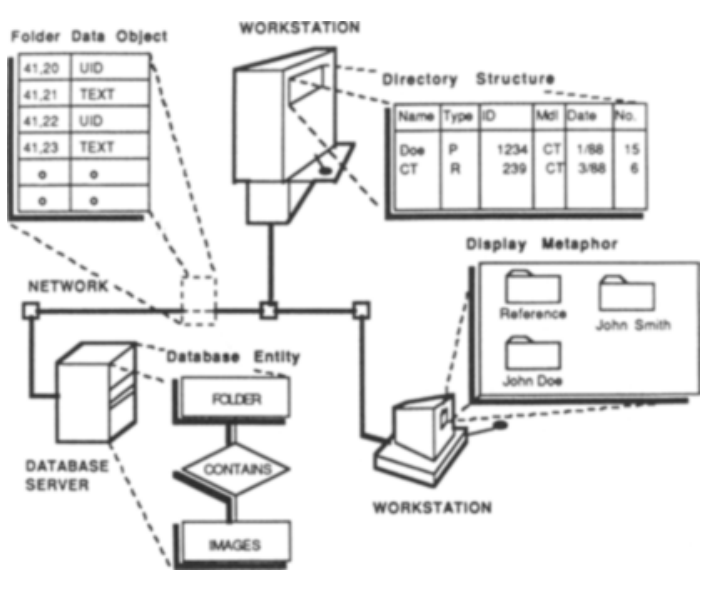

Fig 2. Incarnations of the folder concept.

data objects, the folder has no image data or other data. Instead, its "data" comprise a list of UIDs and textual descriptions; ie, an SPI folder is a data object containing pointers to other data objects, some of which may also be folders.

A data object of type folder may be communicated over a network just as any other data object. It permits one device to describe an association among a set of data objects, and then to communicate that association information to another device. Because the association information is contained in a separate data object, it is external to the set of objects being associated. The SPI folder is relatively simple. It requires that data objects be taken sequentially as referenced. However, since it is possible to include the UID of one folder data object within another, complex associations may be described. By including a folder type element within the DESC of the folder, it is possible to define special purpose data structures, eg, reference folders, examination folders, and history folders.

Communicating association information is only part of the solution to the disparate directory problem. A mechanism is required to normalize the various directory structures of imaging modalities into a generic PACS directory. The final incarnation of the folder concept provides the key. A PACS directory database can be structured as a set of folder entities. Physically, each folder entity is a table in the database. The association information communicated by folder data objects can be encoded as relations between database entities. The following section describes in detail such a database structure.

\section{IMS DATABASE SCHEMA}

A set of entities have been identified for an IMS database that should have meaning to clinicians, who wish to locate a particular set of related data objects. It is assumed that these entities are also sufficiently general to permit the organization of data objects from different modalities. The key set of entities involved are: Patient, Master Jacket, Hospitalization Folder, History Folder, RIS/demographics Folder, and Examination Folder. Figure 3 is an Entity-Relationship Model $^{7}$ that illustrates a schema for an IMS database based on these entities.

The patient entity is relatively straightforward. Each patient must have a unique identifier that will be used as a key (eg, a combination of patient and institution identification code could be used). This list of patients must be maintained to provide access to the patient's Master Jacket which could be saved on-line or archived (see Infinite Database Problem).

By analogy to a film-based radiology department, each patient has a Master Jacket. This can be viewed as a folder that contains a complete clinical history of a patient. It contains three major entities: a radiology information system (RIS) folder, a history folder, and one or more patient hospitalization folders. The RIS folder contains demographic data of a patient. This entity has attributes that identify and describe the patient. These attributes are assumed to be relatively constant over time. The values for the attributes of the RIS folder will be derived from a PACS-RIS interface, or in the absence of a

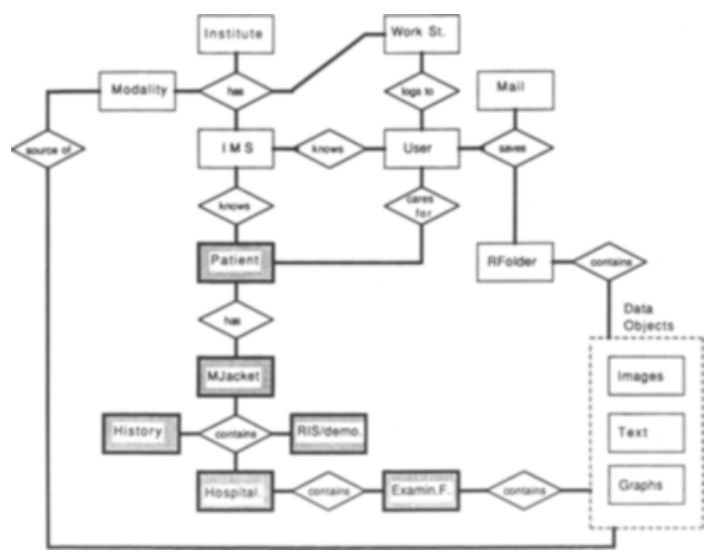

Fig 3. Information management system entity-relationship model. 
RIS, from a PACS administrative node. The history folder contains a history of the patient's diagnosis, as entered by the attending physician or radiologist, as well as selected images and reports.

The need for a hospitalization folder is perhaps not immediately obvious. Over a period of years, a given patient may be treated at an institution a number of times, either as an inpatient or outpatient. Each treatment session, or encounter is defined as a hospitalization. This is an idea common to many RISes and helps to identify current records. There are also many attributes of the patient (eg, referring physician, attending physician, admitting diagnosis, etc) that change with each hospitalization. It is assumed that values for attributes of the hospitalization folder will be derived from a PACS-RIS interface.

The entity of particular importance for organizing data objects is the examination folder. The examination folder, in this context, may be defined as a set of related data objects generated during one contiguous period of time at a particular imaging station. The principle use of the examination folder is to organize images that result from a single study, however, as was previously noted, the association rules that may be used to define a folder are modality- and site-specific. For any hospitalization, a patient may have one or more examinations. Each examination consists of at least one data object (eg, Image, Report, Graph etc.).

The remaining entities and relationships illustrated in Fig 3 define access control mechanisms, user services, and alternative data object access paths. These structures have been included for completeness, but will not be considered in detail.

\section{THE INFINITE DATABASE PROBLEM}

A PACS database server such as the IMS, has a fundamental problem. It is essentially openended. Without some rule for when to delete a patient's records, the database grows indefinitely. Even with a mechanism to remove obsolete records, the database can grow quite large, given estimated image generation rates and legal requirements for storage duration. Having several Terabytes of information online would not only be expensive to maintain, but would impact the performance of the IMS. It is essential to find some mechanism to compress and decompress patient information (the issue is not image compression, but database compression).

One possible solution is to make additional use of the folder concept. Three IMS Database phases may be identified: Active, Inactive, and Archived. Figure 4 illustrates the granularity of the data, at the various phases. The duration of the Active and Inactive databases should be site-configurable. The Archived database is permanently stored on optical media. The Active database maintains a patient's data as database entities up to the Examination Folder level. After a predefined period of time, these data are compressed into the patient's Master Jacket, which is then transferred to the Inactive database. The patient's Master Jacket remains in the Inactive database as a database entity. One can address queries to both the Active and Inactive databases, since the data is in the form of database entities. Again, after a predefined period of time, the patient's Master Jacket can be further compressed as a folder data object and archived. References to the patient's folder data object are maintained in the Active database along with the patient's demographic data. Should there be a need to access a patient's archived Master Jacket, a decompression routine will reinsert the patient's entities into the Active database. It is assumed that decompression will normally be triggered by the readmission of the patient, and so could be performed as a background operation.

\section{CONCLUSIONS}

Considerable work has gone into the design of PACS workstations and communication inter-

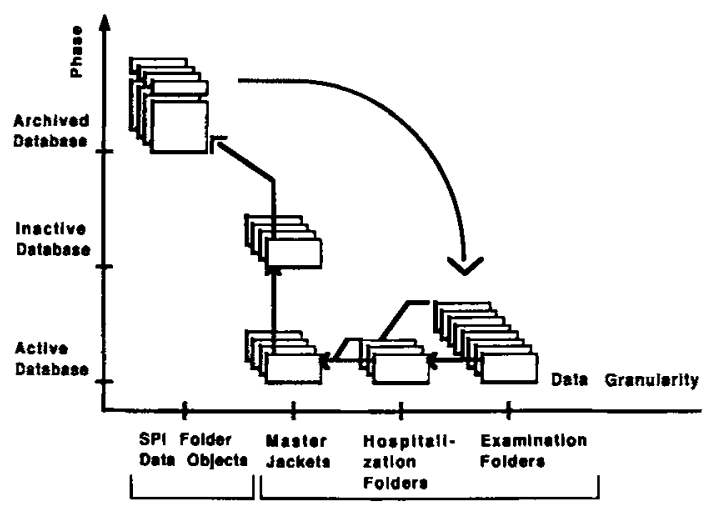

Fig 4. Phase $v$ granularity in an IMS database. 
faces, but issues of data organization in storage components are just beginning to be considered. In this article several important design problems have been identified, including the need for: (1) a unique data object identifier, (2) a mechanism to communicate association rules, (3) a generic PACS directory structure, and (4) a mechanism to compress and decompress PACS databases. The folder concept has been shown to be a powerful tool to address these issues.

If one defines a UID, it is possible and advantageous to design a system architecture that includes distributed file servers and a centralized directory database server. The unique id also permits the definition of a data object of type folder that may be used to communicate association information between nodes. By expanding the folder concept to include database entities, it is possible to design a schema for a PACS database server that simulates the data organization currently used in radiology. By the use of both the folder as database entity and as data object, it is possible to deal with the potentially massive growth potential of PACS databases.

For the filmless radiology department to become a reality, additional work is required in the areas of information storage and retrieval. PACS is somewhat unique in its potential for amassing huge quantities of digital data. Effective management of these data is the key to success.

\section{REFERENCES}

1. Uniformed Services University of the Health Sciences The DIN Report: Functional Requirements for a HospitalBased Digital Imaging Network and Picture Archiving and Communication System. Prepared for USUHS by Mitre Corp, Mclean, VA, Mitre Corporation, 1985

2. Cox GC, Dwyer SJ III, Templeton AW: Computer networks for image management in radiology: An overview. CRC Crit Rev Diagn Imaging 25:333-371, 1986

3. Date CJ: Database Systems, vol 1. Reading, MA, Addison-Wesley, 1986

4. National Electrical Manufacturers Association: ACRNEMA Digital Imaging and Communications Standard.
Washington, DC, National Electrical Manufacturers Association, 1985

5. Siemens AG: Standard Product Interconnect (SPI) for Compatibility of Digital Imaging. Erlangen, FRG, AG Siemens, 1987

6. Greinacher CFC, Fuchs D, Perry J: Related Advantages of a Structured PACS Architecture. Erlangen, West Germany, Siemens AG, Medical Engineering Group, 1986

7. Chen, PPS: Database Design Based on Entity and Relationship, in Yao SB (ed): Principles of Database Design, Englewood Cliffs, NJ, Prentice-Hall, 1985, pp 174-210 